\title{
5-Arylideneimidazolones as a potential solution for multi-drug resistance in cancer cells
}

MEDICAL

COLLEGE

\section{Aneta Kaczor ${ }^{1}$, Nikoletta Szemerédi ${ }^{2}$,Monika Dąbrowska ${ }^{3}$,}

Małgorzata Starek ${ }^{3}$, Gabriella Spengler ${ }^{2}$, Jadwiga Handzlik ${ }^{1}$

${ }^{1}$ Department of Technology and Biotechnology of Drugs, Faculty of Pharmacy, Jagiellonian University Medical College, 9 Medyczna Street, 30-688 Kraków, Poland

${ }^{2}$ Department of Medical Microbiology and Immunobiology, Faculty of Medicine, University of Szeged, Dóm tér 10, H-6720 Szeged, Hungary

${ }^{3}$ Department of Inorganic and Analytical Chemistry, Faculty of Pharmacy, Jagiellonian University Medical College, 9 Medyczna Street, 30-688 Kraków, Poland

\section{Introduction}

One of a serious threats in treatment of cancer diseases is multi-drug resistance (MDR). MDR of cancer cells is a significant problem of chemotherapy failure and is growing rapidly. Thus, searching for compounds able to block at least one mechanism of cancer MDR is an important goal of medicinal chemistry [1]. Such activity was described previously for imidazolones e.g. 5-arylideneimidazolone presented in Fig. 1 [2].

\section{Synthesis}

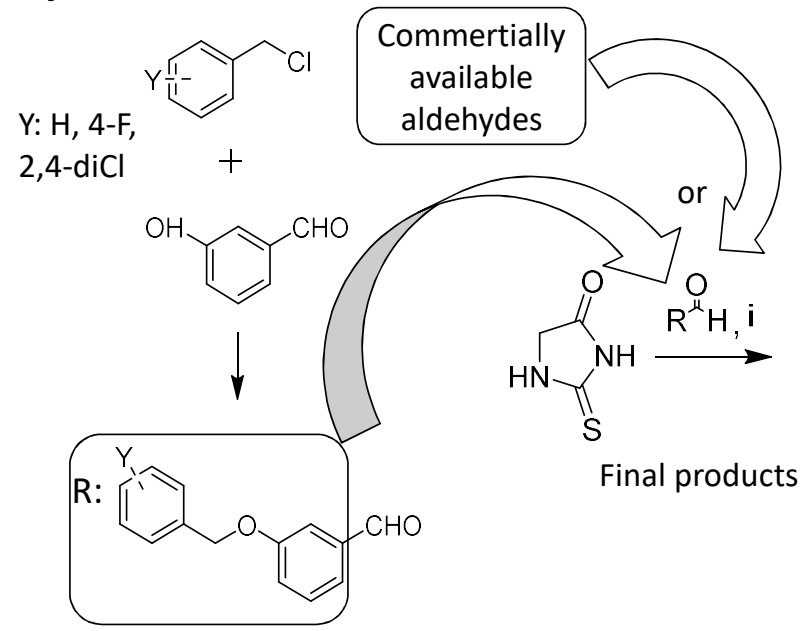

(i) Knoevenagel condensation

(ii) S-methylation

(iii) Reaction with amine

(iv) Dimroth rearrangement

Figure 1. Previously found active compound [2].

MDR in cancer cells in the group of 5-arylideneimidazolones.

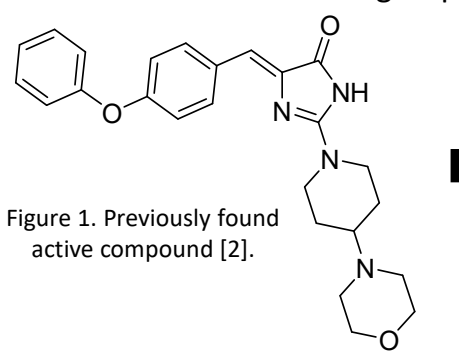

Figure 2. General structure.
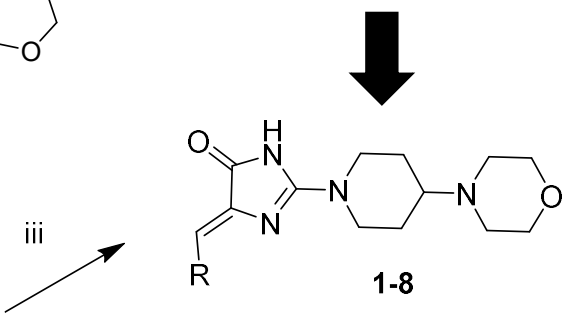

$1-8$<smiles>[R]C=C1NC(=S)NC1=O</smiles><smiles></smiles>

\section{Accumulation assay}

Final compounds were tested in rhodamine 123 accumulation assay using both, sensitive and Pgp overexpressing MDR, mouse T-lymphoma cell lines. Results for the most active compounds in MDR cancer cells in tested concentrations are presented in Table 1.

Table 1. Active compounds 6, 8, 16, 18 in MDR cancer cells.

\begin{tabular}{|c|c|c|}
\hline \multirow{2}{*}{ Compound } & \multicolumn{2}{|c|}{ FAR } \\
\cline { 2 - 3 } & $2 \mu \mathrm{M}$ concentration & $20 \mu \mathrm{M}$ concentration \\
\hline 6 & $\mathbf{1 5 . 1 4 7}$ & $\mathbf{2 8 . 2 6 9}$ \\
\hline 8 & 1.952 & 5.976 \\
\hline 16 & 2.224 & $\mathbf{1 1 . 3 3 1}$ \\
\hline 18 & 15.807 & $\mathbf{3 0 . 8 6 0}$ \\
\hline Tariquidar & - & $\mathbf{3 4 . 8 0 9}$ \\
\hline
\end{tabular}

\section{Cytotoxicity toward cancer cells}

Table 2. The most cytotoxic compounds from this group.

\begin{tabular}{|c|c|c|c|c|}
\hline \multirow{2}{*}{ Compounds } & \multicolumn{2}{|c|}{ PAR $\left(\mathrm{IC}_{\mathbf{5 0}} \mu \mathrm{M}\right)$} & \multicolumn{2}{c|}{ MDR $\left(\mathrm{IC}_{\mathbf{5 0}} \boldsymbol{\mu M}\right)$} \\
\cline { 2 - 5 } & Mean & SD & Mean & SD \\
\hline $\mathbf{9}$ & $>100$ & - & $\mathbf{2 , 1 5}$ & 0,03 \\
\hline $\mathbf{1 0}$ & $>100$ & - & $\mathbf{2 , 3 7}$ & 0,11 \\
\hline
\end{tabular}

\section{Lipophilicity}

RP-TLC method

All tested compounds proved "drug-like" lipophilicity $\left(\mathrm{R}_{\mathrm{MO}}=1.48-3.35\right)$

\section{Conclusions}

Two of tested 5-arylideneimidazolones (6 and 18):

$\checkmark$ displayed strong activity in MDR cancer cells

$\checkmark$ did not show cytotoxicity,

$\checkmark$ proved „drug-like" lipophilicity.
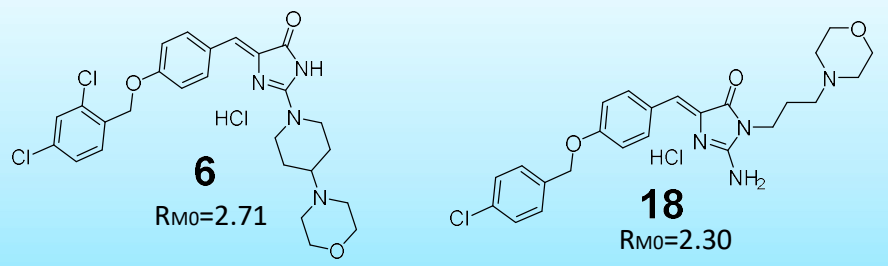

\section{Bibliography}

[1] Szakács G. et al. Nature Reviews Drug Discovery 5 (2006) 219. [2] Kaczor A. et al. Molecules 25 (2020) 2258.

\section{Acknowlegments}

This work was supported by grant from Ministry of Science and Higher Education budget funds for science in 2017-2020, as a research project within "Diamond Grant" no 0169/DIA/2017/46 and Jagiellonian University Medical College grants (N42/DBS/000070 and N42/DBS/000027). 